\title{
Triptolide exerts pro-apoptotic and cell cycle arrest activity on drug-resistant human lung cancer A549/Taxol cells via modulation of MAPK and PI3K/Akt signaling pathways
}

\author{
CHEN QIONG XIE ${ }^{1}$, PING ZHOU $^{1}$, JIAN ZUO $^{1}$, XIANG LI $^{1,2}$, YONG CHEN $^{1}$ and JIAN WEI CHEN ${ }^{1,3}$ \\ ${ }^{1}$ College of Pharmacy; ${ }^{2}$ Jiangsu Key Laboratory for Chinese Material Medical Processing; \\ ${ }^{3}$ Jiangsu Key Laboratory for TCM Formulae Research, Nanjing University of Chinese Medicine, \\ Nanjing, Jiangsu 210046, P.R. China
}

Received June 29, 2015; Accepted August 9, 2016

DOI: $10.3892 / \mathrm{ol} .2016 .5099$

\begin{abstract}
Multidrug resistance (MDR) is a major obstacle in the effective chemotherapeutic treatment of cancers. Triptolide (TPL) is a diterpenoid isolated from Tripterygium wilfordii Hook. f., a traditional Chinese medicine. It was demonstrated in our previous study that TPL exerts anti-MDR cancers on various MDR cell lines (including A549/Taxol, MCF-7/ADR and Bel7402/5-Fu). The present study was designed to investigate its anti-proliferative activity on A549/Taxol cells, and explore the underlying mechanism of action. The anti-proliferative activity of TPL on A549/Taxol cells was assessed by 3-(4,5-dimethylthiazol-2-yl)-2,5-diphenyltetrazolium bromide (MTT) assay. Its pro-apoptosis and cell cycle arrest activities were analyzed by flow cytometry. Western blot assay was employed to investigate the levels of mitogen-activated protein kinases (MAPKs) and apoptosis-related proteins in cells. TPL efficiently suppressed the proliferation of A549/Taxol cells. Co-treatment with MAPK inhibitors in the MTT assay indicated that the extracellular signal-regulated kinase (ERK) and c-Jun N-terminal kinase (JNK) pathways were involved in the process. Upregulation of p-p38, p-ERK, p-GSK-3 $\beta$, Bax and cleaved caspases-3 and -9, and downregulation of p-JNK, p-Akt and Bcl-2 were observed upon treatment with TPL in the A549/Taxol cells. The results from flow cytometry assay revealed that TPL induced apoptosis and S-phase arrest in A549/Taxol cells. This occurred as a result of the upregulation of p-ERK and p-GSK-3 $\beta$, and the downregulation of $\mathrm{p}$-JNK and $\mathrm{p}$-Akt, and was responsible for the subsequent anti-proliferative activity.
\end{abstract}

Correspondence to: Professor Xiang Li or Dr Yong Chen, College of Pharmacy, Nanjing University of Chinese Medicine, 138 Xian Lin Road, Nanjing, Jiangsu 210046, P.R. China

E-mail: lixiang_8182@163.com

E-mail: achenyongmail@163.com

Key words: multidrug resistance, triptolide, apoptosis, S-phase arrest, mitogen-activated protein kinases, PI3K/Akt

\section{Introduction}

Lung cancer is the most commonly diagnosed cancer worldwide, contributing to $12.7 \%$ of the total incidence of cancers, and also the leading cause of mortality among all tumors (18.2\% of the total) (1). Multidrug resistance (MDR) is a phenomenon whereby cancer cells exhibit simultaneous resistance to anti-cancer drugs, with various structures and mechanisms of action $(2,3)$. MDR results in poor therapeutic efficacy in the later stage of cancer treatments, and it is also the main obstacle in obtaining a satisfactory therapeutic outcome in lung cancer (4).

Extensive studies have revealed that MDR cancer cells are immune from anti-cancer drug-induced cell apoptosis through the upregulation of survival signaling pathways, including phosphatidyl-3-phosphate kinase (PI3K) and extracellular-regulated kinase-1 (ERK1) (5), or the suppression of anti-proliferative signaling pathways, including p38 mitogen-activated protein kinases (MAPKs) (6). Bcl-2 is an oncogene which contributes to tumor occurrence mainly due to the inhibition of apoptosis, and the overexpression of Bcl-2 usually results in the resistance of cancer cells to anti-cancer agents associated with abnormal changes of the pathways discussed above $(7,8)$.

Preliminary observations by the present authors demonstrated that the pro-apoptotic and cell cycle arrest activities of triptolide (TPL) largely contribute to its antitumor effect, and were direct outcomes of the modulation of various important upstream pathways, including PI3K/Akt, MAPK, JAK/STAT and nuclear factor- $\kappa \mathrm{B}$, which subsequently modulated the expression of apoptosis-related proteins such as those of the Bcl-2 family (9-11). In our preliminary observations, we demonstrated that TPL inhibited the proliferation of MDR A549/Taxol cells in vitro mainly through selective modulation of MAPK signaling (9-11). However, until now, our understanding of the association between the inhibitory activity of TPL in MDR cells and the MAPK pathway has been limited. Hence, in the present study, we investigated the modulatory effect of TPL on the MAPK pathway in A549/Taxol cells along with its correlation with the inhibitory effect on the proliferation. 


\section{Materials and methods}

Drugs and reagents. TPL ( $\geq 98 \%$, L-004-130304) was purchased from Chengdu Herb Purity Co., Ltd. (Chengdu, China). It was dissolved in dimethyl sulfoxide (DMSO) at a concentration of $10 \mathrm{mmol} / 1$ to obtain the stock solution and kept below $-20^{\circ} \mathrm{C}$, then diluted to various concentrations with phosphate-buffered saline (PBS) prior to the assays. The final concentration of DMSO was less than 0.1\%. 3-(4,5-dimethylthiazol-2-yl)-2,5-diphenyltetrazolium bromide (MTT), RPMI-1640, a bicinchoninic acid (BCA) protein assay kit, inhibitor of ERK U0126, propidium iodide (PI) staining kit and Annexin V-fluorescein isothiocyanate (FITC)/PI apoptosis detection kit were purchased from Nanjing KeyGen Biotechnology Co. Ltd. (Nanjing, China). Anti-p-p38, anti-p-JNK, anti-p-ERK antibodies, inhibitor of p38 SB202190 and inhibitor of JNK SP600125 were purchased from Cell Signaling Technology (Beverly, MA, USA). Anti-p-Akt (Ser473), anti-p-GSK-3 $\beta$ (Ser9), cleaved caspase (c-caspase)-3 (1:200), c-caspase-9 (1:1,000), Bcl-2 (1:500) and COX IV $(1: 1,000)$ were supplied by Bioworld Technology (Nanjing, China). An enhanced chemiluminescence kit was purchased from Thermo Fisher (Shanghai, China). Bovine calf serum was purchased from Wisent corporation (Nanjing, China). All other chemicals and reagents used were of analytical grade.

Cell culture. The human lung cancer cell line A549 and the corresponding MDR cell line A549/Taxol were purchased from Nanjing KeyGen Biotechnology Co. Ltd., and cultured in RPMI-1640 medium supplemented with $10 \%$ bovine calf serum, $100 \mathrm{U} / \mathrm{ml}$ penicillin and $100 \mu \mathrm{g} / \mathrm{ml}$ streptomycin at $37^{\circ} \mathrm{C}$ in a humidified atmosphere of $5 \% \mathrm{CO}_{2}$. Paclitaxel (Taxol, $200 \mathrm{ng} / \mathrm{ml}$ ) was added to the medium in order to maintain MDR in the A549/Taxol cells, and removed two weeks before the experiments. Cells were passaged every 2-3 days.

Cell viability. For the cell viability assay, A549 and A549/Taxol cells were seeded onto a 96-well plate at a density of $8 \times 10^{3}$ cells per well. Following overnight incubation, the culture medium was aspirated, and the cells were incubated with various concentrations of TPL (the final concentrations were $0.01,0.02,0.04,0.06$ and $0.08 \mu \mathrm{mol} / \mathrm{l}$ ) or co-treated with MAPK inhibitors in complete culture medium for $48 \mathrm{~h}$. The same volume of complete culture medium served as the negative control. Then $20 \mu \mathrm{l}$ MTT solution $(5 \mathrm{mg} / \mathrm{ml})$ was added to each well, and the plates were further incubated for $4 \mathrm{~h}$. The medium was removed and $150 \mu 1$ DMSO was added to solubilize the MTT formazan salt. The absorbance of the solution was measured on a microplate reader (Spectra MAX190, Molecular Devices LLC, Sunnyvale, CA, USA) at $490 \mathrm{~nm}$ and the results were expressed as a percentage of the control cells.

Apoptosis and cell cycle analyses. A549/Taxol cells were seeded onto six-well plates at a density of $3 \times 10^{5}$ cells per well. Following overnight incubation, cells were treated with TPL at concentrations of 0.025 and $0.05 \mu \mathrm{M}$ for $24 \mathrm{~h}$. For the purpose of apoptosis analysis, following treatment with TPL, the cells were harvested and washed with PBS, and then stained with Annexin V/PI according to the manufacturer's recommendations. Stained samples were analyzed by flow cytometry
(FACSCalibur, BD Biosciences, San Jose, CA, USA). For analysis of the cell cycle distribution, the supernatant was discarded, and attached cells were harvested and fixed in cold $70 \%$ ethanol overnight at $-20^{\circ} \mathrm{C}$. Cell cycle distribution was analyzed using a flow cytometer according to the manufacturer's instructions for the PI staining kit.

Protein extraction and western blot. A549/Taxol cells ( $3 \times 10^{5}$ cells/well) were treated with TPL at concentrations of 0.025 and $0.05 \mu \mathrm{M}$, or co-treated with MAPK inhibitors, for $24 \mathrm{~h}$. Following the treatments, cells were collected, washed twice with pre-chilled PBS, lysed, and then centrifuged at $12,000 \mathrm{rpm}$ for $10 \mathrm{~min}$ at $4^{\circ} \mathrm{C}$. The supernatant of the lysate was boiled, and total protein was measured using the BCA protein assay kit. Proteins were separated by SDS-PAGE, and then transferred onto polyvinylidene fluoride (PVDF) membranes ( $0.45 \mu \mathrm{m}$; Millipore). Non-specific protein binding sites were blocked with $5 \%$ bovine serum albumin diluted in Tris-buffered saline buffer containing 0.1\% Tween-20 (pH 7.4) for $2 \mathrm{~h}$ at room temperature, followed by incubation with the appropriate primary and secondary antibodies. The expression of proteins was detected using a Chemi Doc XRS+ system (Bio-Rad Laboratories, Inc., Hercules, CA, USA). The grey level ratio of the target protein to COX IV was used to represent the relative protein expression level.

Statistical analysis. Results are expressed as the means \pm standard deviation. Statistical differences among groups were evaluated by the t-test using GraphPad Prism 6. $\mathrm{P}<0.05$ and $\mathrm{P}<0.01$ were considered to represent different levels of statistical significance.

\section{Results}

Resistance fold of A549/Taxol cells. A549 and A549/Taxol cells were treated with Taxol for $48 \mathrm{~h}$ and the cytotoxicity was assessed using MTT assay. The $\mathrm{IC}_{50}$ of Taxol against A549 and A549/Taxol cells was 3.52 \pm 0.47 and $71.31 \pm 7.95 \mu \mathrm{M}$, respectively. A549/Taxol cells exhibited more than a 20 -fold resistance to Taxol in comparison with the drug-sensitive A549 cell line.

TPL inhibits proliferation of A549/Taxol cells. Various doses $(0.01,0.02,0.04,0.06,0.08 \mu \mathrm{M})$ of TPL were used to treat A549/Taxol cells for $48 \mathrm{~h}$. The anti-proliferative effect of TPL on A549/Taxol was assessed by MTT assay, and the results revealed that TPL inhibited the proliferation of A549/Taxol cells efficiently in a dose-dependent manner, with an $\mathrm{IC}_{50}$ value of $46.47 \pm 0.31 \mathrm{nM}$. It was far more efficient than the positive drug cisplatin, which demonstrated an $\mathrm{IC}_{50}$ value of $8.87 \pm 0.98 \mathrm{uM}$. TPL exhibits potent cytotoxicity in vitro in A549/Taxol cells. Based on the primary findings, we used TPL at concentrations of 0.025 and $0.05 \mu \mathrm{M}$ in subsequent experiments.

TPL modulates MAPKs in A549/Taxol cells. To investigate the modulatory effects of TPL on MAPKs in the course of inhibition of proliferation of A549/Taxol cells, we performed MTT assay using MAPK inhibitors (SB202190, SP600125 and U0126) coupled with TPL. SB202190 exerted little effect 

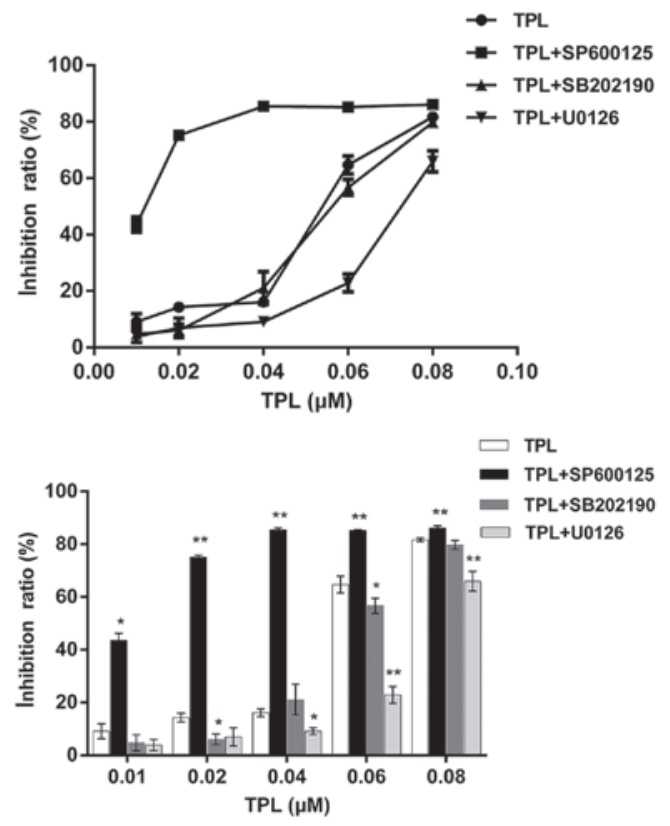

Figure 1. Inhibition of triptolide (TPL) and mitogen-activated protein kinase inhibitors on proliferation of A549/Taxol cells. TPL inhibited the proliferation of A549/Taxol cells in a concentration-dependent manner. SP600125 reinforced the effect, particularly when TPL was at low concentrations, while U0126 attenuated the activity. SB202190 had little effect on the results Results were assessed by 3-(4,5-dimethylthiazol-2-yl)-2,5-diphenyltetrazolium bromide (MTT) assay, and values are presented as means \pm standard deviation, $\mathrm{n}=3$. ${ }^{* * *} \mathrm{P}<0.01$ and ${ }^{*} \mathrm{P}<0.05$, compared with the corresponding TPL group at the same concentration.

on the result, while SP600125 and U0126 significantly reinforced the inhibitory effects of TPL (Fig. 1). Since there was no significant effect observed with the addition of SB202190, it was suggested that the modulation of $\mathrm{p} 38$ contributed little to the inhibitory effect exhibited by TPL on A549/Taxol cells. SP600125 exhibited synergistic effects with TPL, while antagonism caused by U0126 was also noted. These findings suggested that TPL may exert its inhibitory effects by regulating the JNK and ERK signaling pathways.

TPL exerts anti-proliferative effects on A549/Taxol cells via pro-apoptosis. To investigate the mechanism of TPL's involvement in the anti-proliferative activity of A549/Taxol cells, A549/Taxol cells were treated with TPL at concentrations of 0.025 and $0.05 \mu \mathrm{M}$. Twenty-four hours later, Annexin V-FITC/PI staining flow cytometry was used to assess the rate of cell apoptosis. Compared with the control group, we observed that the number of apoptotic cells increased significantly in the TPL group (Fig. 2A). In addition, we noted that TPL significantly increased the expression of caspase- 3 and caspase-9 (Fig. 2B). These results indicated that TPL induced caspase family-dependent apoptosis in A549/Taxol cells. In addition, as shown in Fig. 2B, upregulation of the Bax/Bcl-2 ratio was observed following TPL treatment, which supported our previous hypothesis concerning the role of pro-apoptosis.

Induction of S-phase arrest in A549/Taxol cells by TPL. To elucidate the mechanism of TPL-induced proliferation inhibition, we examined the effect of TPL on cell phase distribution by flow cytometry. As shown in Fig. 3, concomitant with the

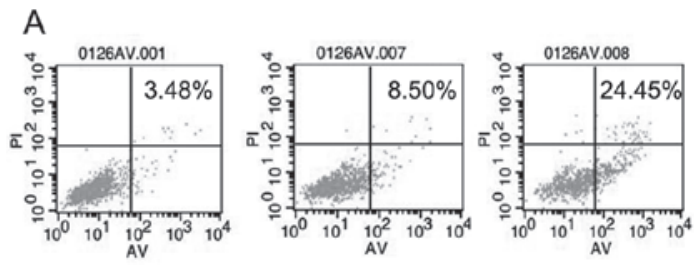

B

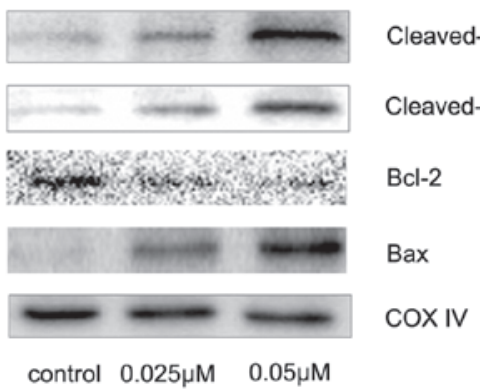

Figure 2. (A) Triptolide (TPL) induces apoptosis of A549/Taxol cells in a concentration-dependent manner. (B) TPL induces apoptosis through upregulation of cleaved caspase-3/-9 and Bax, and downregulation of Bcl-2.
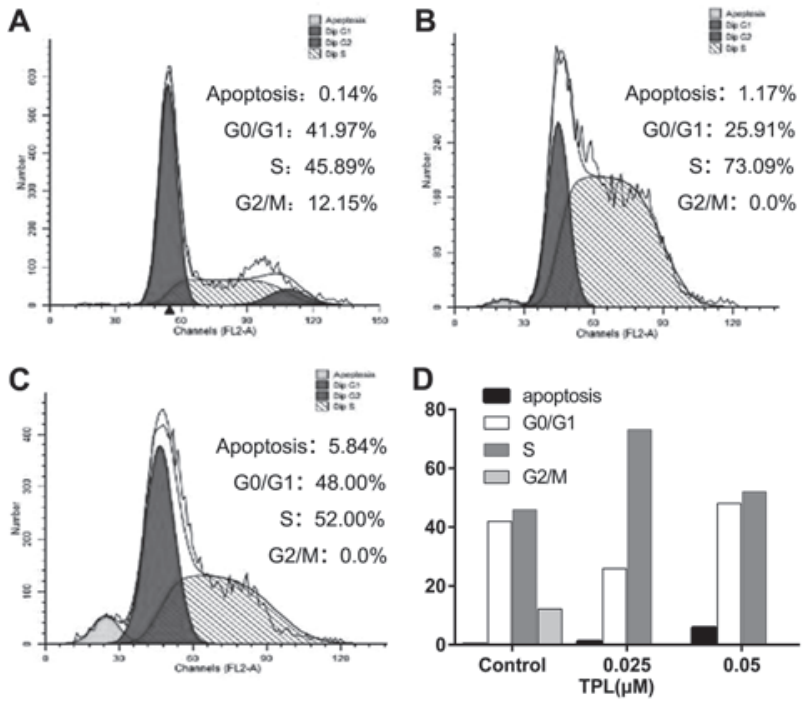

Figure 3. Triptolide (TPL) induces S-phase arrest in A549/Taxol cells. The representative cell cycle distribution of (A) A549/Taxol cells without TPL treatment and (B and C) A549/Taxol cells treated with 0.025 and $0.05 \mu \mathrm{M}$ TPL are shown using flow cytometry. (D) The proportion of TPL-treated A549/Taxol cells in the S-phase was significantly increased, with a concomitant reduction in the proportion of cells in the $\mathrm{G}_{2} / \mathrm{M}$ phase when compared with the control.

growth inhibitory effect, treatment with TPL induced a significant S-phase arrest. The cell populations in the $\mathrm{G}_{0} / \mathrm{G}_{1}, \mathrm{~S}$ and $\mathrm{G}_{2} / \mathrm{M}$ phases were $41.97,45.89$ and $12.15 \%$ in the control group. However, after 24-h incubation with 0.025 and $0.05 \mu \mathrm{M}$ TPL, the S-phase portion was notably enhanced by 27.2 and $6.11 \%$, respectively. Contrarily, the $\mathrm{G}_{2} / \mathrm{M}$ population of A549/Taxol was markedly reduced following treatment with 0.025 and $0.05 \mu \mathrm{M}$ TPL, indicating the blockage of the $\mathrm{S}_{-} \mathrm{G}_{2}$ transition in A549/Taxol cells. In addition, the sub- $\mathrm{G}_{1}$ population was increased by 1.03 and $5.70 \%$, respectively.

Measurement of MAPKs and PI3K/Akt signaling pathways. The inhibition of p38 usually promotes tumor formation, but in 


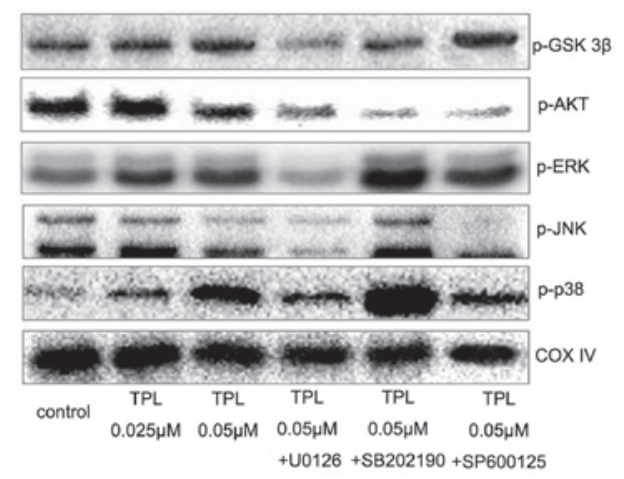

Figure 4. Triptolide (TPL) and mitogen-activated protein kinase inhibitors modulate the expression of p-GSK-3 $\beta$, p-Akt, p-ERK, p-JNK and p-p38 in A549/Taxol cells following $24 \mathrm{~h}$ treatment. TPL upregulated $\mathrm{p}-\mathrm{ERK}$ and p-p38 in a concentration-dependent manner, while it downregulated p-JNK.

certain cases, the tumor-forming ability is suppressed by p38 activation. JNK plays two antagonistic roles: oncogenic and and pro-apoptotic. The oncogenic function of JNK pathways is highly expressed in cancer cells, which promotes cell proliferation $(12,13)$. The ERK1/2 cascades are mainly involved in tumor cell survival and proliferation regulation, but increased levels of the phosphorylated form of ERK1/2 also induce cell apoptosis (14). The aberrant activation of Akt is one of the most frequent alterations observed in most types of cancer cells, and it has been observed to correlate with poor prognosis and resistance to various anti-cancer agents (15).

Preliminary observations in the current study shed light on the mechanism of action of TPL on the inhibition of proliferation of A549/Taxol cells. Based on the findings obtained, we proposed that modulation of JNK and ERK signaling by TPL contributed in a great manner to the anti-proliferative activity observed. To validate the conclusion, the expression of three MAPKs (p-p38, p-JNK and p-ERK) and PI3K/Akt (p-Akt and p-GSK-3 $\beta$ ) was analyzed by western blot analysis. As shown in Fig. 4, TPL increased the expressions of p-p38, p-ERK and p-GSK-3 $\beta$, but exerted the opposite effect on p-JNK and p-Akt. Compared with the TPL group, the expression of p-ERK, p-JNK and p-Akt was notably downregulated by MAPK inhibitors, while the $\mathrm{p}-\mathrm{GSK}-3 \beta$ expression level was significantly upregulated by SP600125. These results were consistent with conclusions deduced from the results of MTT assay.

\section{Discussion}

MDR is a major obstacle in effective cancer chemotherapy. Following cycles of chemotherapy, cancer cells have been demonstrated to form simultaneous resistance to anti-cancer drugs with various structures and mechanisms of action $(2,3)$. Traditional Chinese medicines have numerous advantages over chemical agents, including low cost and lower toxicity, and have long been used in the clinical treatment of cancer. In previous studies, researchers have paid increasing attention to Chinese medicines in their search for new potential MDR reversal agents (16).

TPL is a diterpenoid triepoxide isolated from the traditional Chinese herb Tripterygium wilfordii Hook. f. It possesses efficient antitumor activity against a range of cancer cells. TPL has been demonstrated to exert its reversal activity on various MDR cancer cells, mainly through the downregulation of MDR proteins, including P-glycoprotein (17-19). Our primary study revealed that TPL could notably inhibit the proliferation of a panel of MDR cancer cell lines, including A549/Taxol, MCF-7/ADR and Bel7402/5-Fu (20). Significantly, we observed that the activity was mainly associated with the modulation of MAPKs and the downstream pathways. Although previous studies provided useful clues for a better understanding of the mechanism concerning the inhibition of proliferation of MDR cells mediated by TPL, there is insufficient evidence to characterize the exact role of MAPKs in this process. Hence, we designed and carried out the present study.

MAPKs play significant roles in regulating an array of cellular responses, particularly those involving cell proliferation and apoptosis, and the modulation of these kinases may bring promising benefits to the therapeutic treatment of tumors (21-23). The development of MDR is often accompanied by the modulation of MAPKs (24-26). ERK1 and 2 are known to act as anti-apoptotic cascades by transducing survival signals, whereas JNK or p38 phosphorylation results in the promotion of apoptosis (27). However, the roles of these pathways are sophisticated. For example, suppression of the MEK-ERK signaling pathway by PD98059 increased rather than decreased cisplatin resistance (28). In addition, inhibition of p38 MAPK significantly reduced gemcitabine sensitivity in NTUB1 cells, and Calebin-A enhanced the cytotoxicity of vincristine in SGC7901/vincristine cells through the inhibition of JNK $(29,30)$. The exact effect of the modulation of MAPKs depends on the cell types and crosstalk among the various pathways. The results from this study clearly demonstrate that TPL exerts its anti-proliferative effect via downregulation of p-JNK and upregulation of the p-ERK and p-p38 pathways. The selective modulation effects differed from the results of other studies, and are worthy of further investigation.

PI3K/Akt has emerged as an essential pathway in regulating cell proliferation, survival and apoptosis, and cell migration. Accumulating evidence reveals that Akt activation plays a significant role in the chemoresistance of cancer cells $(12,31)$. Activation of the Akt signaling pathway mediates acquired resistance to sorafenib in hepatocellular carcinoma cells (32), and confers resistance to gefitinib in lung cancer cells (33). Akt phosphorylation entails inhibition of a highly conserved GSK-3, and GSK-3 $\beta$ inactivation leads to $\beta$-catenin accumulation, which entails drug resistance (34). Consistent with these studies, downregulation of $\mathrm{p}$-Akt and further regulation of the downstream signal $\mathrm{p}-\mathrm{GSK}-3 \beta$ results in anti-proliferation of TPL in A549/Taxol cells. SP600125 increased the expression of p-GSK-3 $\beta$ and reinforced the effect of TPL, whereas U0126 downregulates the expression of $p-G S K-3 \beta$. These findings support the hypothesis that modulation of TPL in the PI3K/Akt signaling pathway contributed to the pro-apoptosis and the subsequent anti-proliferation of A549/Taxol cells, and that the effect was regulated by the modulation effect of TPL on ERK. MAPKs and PI3K/Akt signaling pathways are potential targets of TPL against MDR cancers.

The pro-apoptotic activity of TPL is associated with the modulation of MAPKs, but fundamentally via the modulation of apoptosis-related proteins. The Bcl-2 family plays a key role 
in regulating the intrinsic apoptotic pathway, and is modulated by MAPKs (35). Bax is a pro-apoptotic protein, while Bcl-2 possesses anti-apoptotic properties through stabilizing mitochondrial membrane and suppressing the release of cytochrome $\mathrm{C}$. The balance between the proteins is crucial in the progression of apoptosis (36). Caspases are proteases with a well-defined role in apoptosis, and caspase-3 and - 9 activation eventually results in cell apoptosis. In our study, we observed significant upregulation of Bax and c-caspase-3 and -9, and downregulation of Bcl-2, along with simultaneous modulation of MAPKs following treatment of TPL in A549/Taxol cells. These findings indicate a close association between modulations of $\mathrm{Bcl}$ and MAPKs.

The anti-proliferative activity exerted by TPL on A549/Taxol cells may be due to the modulation of MAPKs and PI3K/Akt pathways, which subsequently led to the activation or suppression of the expression of apoptosis-related proteins, apoptosis and cell cycle arrest. This study provides useful indications for a better understanding of the mechanisms of TPL-induced apoptosis against A549/Taxol cells.

\section{Acknowledgements}

This study was supported by grants from the National Natural Science Fund (81274057 and 81573577) and the Natural Science for Youth Foundation (81403082).

\section{References}

1. Ferlay J, Shin HR, Bray F, Forman D, Mathers C and Parkin DM Estimates of worldwide burden of cancer in 2008: GLOBOCAN 2008. Int J Cancer 127: 2893-2917, 2010.

2. Ling V, Gerlach J and Kartner N: Multidrug resistance. Breast Cancer Res Treat 4: 89-94, 1984.

3. Gottesman MM: Mechanisms of cancer drug resistance. Annu Rev Med 53: 615-627, 2002.

4. Luqmani YA: Mechanisms of drug resistance in cancer chemotherapy. Med Prin Pract 14 (Suppl 1): S35-S48, 2005.

5. Wu G, Qin XQ, Guo JJ, Li TY and Chen JH: AKT/ERK activation is associated with gastric cancer cell resistance to paclitaxel. Int J Clin Exp Pathol 7: 1449-1458, 2014.

6. Olson JM and Hallahan AR: p38 MAP kinase: a convergence point in cancer therapy. Trends Mol Med 10: 125-129, 2004.

7. Adams JM and Cory S: The Bcl-2 protein family: arbiters of cell survival. Science 281: 1322-1326, 1998.

8. Dive C: Avoidance of apoptosis as a mechanism of drug resistance. J Intern Med Suppl 740: 139-145, 1997.

9. Yang M, Huang J, Pan HZ and Jin J: Triptolide overcomes dexamethasone resistance and enhanced PS-341-induced apoptosis via PI3k/Akt/NF-kappaB pathways in human multiple myeloma cells. Int J Mol Med 22: 489-496, 2008

10. Meng GM, Wang W, Chai KQ, Yang SW, Li FQ and Jiang K: Combination treatment with triptolide and hydroxycamptothecin synergistically enhances apoptosis in A549 lung adenocarcinoma cells through PP2A-regulated ERK, p38 MAPKs and Akt signaling pathways. Int J Oncol 46: 1007-1017, 2015.

11. Wang ZP, Jin HF, Xu RD, Mei QB and Fan DM: Triptolide downregulates Rac1 and the JAK/STAT3 pathway and inhibits colitis-related colon cancer progression. Exp Mol Med 41: 717-727, 2009.

12. Wada T and Penninger JM: Mitogen-activated protein kinases in apoptosis regulation. Oncogene 23: 2838-2849, 2004.

13. Bradham C and McClay DR: p38 MAPK in development and cancer. Cell Cycle 5: 824-828, 2006.

14. Modi PK, Komaravelli N, Singh N and Sharma P: Interplay between MEK-ERK signaling, cyclin D1 and cyclin-dependent kinase 5 regulates cell cycle reentry and apoptosis of neurons. Mol Biol Cell 23: 3722-3730, 2012.

15. Huang WC and Hung MC: Induction of Akt activity by chemotherapy confers acquired resistance. J Formos Med Assoc 108: 180-194, 2009.
16. Li C, Sun BQ and Gai XD: Compounds from Chinese herbal medicines as reversal agents for P-glycoprotein-mediated multidrug resistance in tumours. Clin Transl Oncol 16: 593-598, 2014.

17. Guo Q, Nan XX, Yang JR, Yi L, Liang BL, Wei YB, Zhu N, $\mathrm{Hu} \mathrm{SB}$, Zhang H, Luo Y and Xu YF: Triptolide inhibits the multidrug resistance in prostate cancer cells via the downregulation of MDR1 expression. Neoplasma 60: 598-604, 2013.

18. Li H, Hui L, Xu W, Shen H, Chen Q, Long L and Zhu X: Modulation of P-glycoprotein expression by triptolide in adriamycin-resistant K562/A02 cells. Oncol Lett 3: 485-489, 2012.

19. Chen YW, Lin GJ, Chuang YP, Chia WT, Hueng DY, Lin CK, Nieh S and Sytwu HK: Triptolide circumvents drug-resistant effect and enhances 5-fluorouracil antitumor effect on KB cells. Anti-Cancer Drugs 21: 502-513, 2010.

20. Xie CQ, Zhou P, Yuan F, Li X and Chen JW: Screening out potential agents from traditional Chinese medicine with anti-multidrug resistance tumor cells in vitro. Lishizhen Med Mater Med Res 7: 1572-1574, 2015 (In Chinese).

21. Thompson $\mathrm{N}$ and Lyons $\mathrm{J}$ : Recent progress in targeting the Raf/MEK/ERK pathway with inhibitors in cancer drug discovery. Curr Opin Pharmacol 5: 350-356, 2005.

22. Han J and Sun P: The pathways to tumor suppression via route p38. Trends Biochem Sci 32: 364-371, 2007.

23. Bogoyevitch MA, Ngoei KR, Zhao TT, Yeap YY and Ng DC: c-Jun N-terminal kinase (JNK) signaling: recent advances and challenges. Biochim Biophys Acta 1804: 463-475, 2010

24. Katayama K, Yoshioka S, Tsukahara S, Mitsuhashi J and Sugimoto Y: Inhibition of the mitogen-activated protein kinase pathway results in the down-regulation of P-glycoprotein. Mol Cancer Ther 6: 2092-2102, 2007.

25. Barancik M, Bohácová V, Kvackajová J, Hudecová S, Krizanová O and Breier A: SB203580, a specific inhibitor of p38-MAPK pathway, is a new reversal agent of P-glycoprotein-mediated multidrug resistance. Eur J Pharm Sci 14: 29-36, 2001

26. Guo X, Ma N, Wang J, Song J, Bu X, Cheng Y, Sun K, Xiong H, Jiang G, Zhang B, et al: Increased p38-MAPK is responsible for chemotherapy resistance in human gastric cancer cells. BMC Cancer 8: 375, 2008.

27. Krishna $M$ and Narang $H$ : The complexity of mitogen-activated protein kinases (MAPKs) made simple. Cell Mol Life Sci 65: 3525-3544, 2008.

28. Yeh PY, Chuang SE, Yeh KH, Song YC, Ea CK and Cheng AL: Increase of the resistance of human cervical carcinoma cells to cisplatin by inhibition of the MEK to ERK signaling pathway partly via enhancement of anticancer drug-induced NF kappa B activation. Biochem Pharmacol 63: 1423-1430, 2002.

29. Kao YT, Hsu WC, Hu HT, Hsu SH, Lin CS, Chiu CC, Lu CY, Hour TC, Pu YS and Huang AM: Involvement of p38 mitogen-activated protein kinase in acquired gemcitabine-resistant human urothelial carcinoma sublines. Kaohsiung J Med Sci 30: 323-330, 2014.

30. Li Y, Li S, Han Y, Liu J, Zhang J, Li F, Wang Y, Liu X and Yao L: Calebin-A induces apoptosis and modulates MAPK family activity in drug resistant human gastric cancer cells. Eur J Pharmacol 591: 252-258, 2008.

31. Burris HA III: Overcoming acquired resistance to anticancer therapy: focus on the PI3K/AKT/mTOR pathway. Cancer Chemother Pharmacol 71: 829-842, 2013.

32. Chen KF, Chen HL, Tai WT, Feng WC, Hsu CH, Chen PJ and Cheng AL: Activation of phosphatidylinositol 3-kinase/Akt signaling pathway mediates acquired resistance to sorafenib in hepatocellular carcinoma cells. J Pharmacol Exp Ther 337: 155-161, 2011.

33. Liu BN, Yan HQ, Wu X, Pan ZH, Zhu Y, Meng ZW, Zhou QH and $\mathrm{Xu} \mathrm{K}$ : Apoptosis induced by benzyl isothiocyanate in gefitinib-resistant lung cancer cells is associated with Akt/MAPK pathways and generation of reactive oxygen species. Cell Biochem Biophys 66: 81-92, 2013.

34. Galoian K, Temple HT and Galoyan A: mTORC1 inhibition and ECM-cell adhesion-independent drug resistance via PI3K-AKT and PI3K-RAS-MAPK feedback loops. Tumour Biol 33: 885-890, 2012.

35. Frenzel A, Grespi F, Chmelewskij W and Villunger A: Bcl2 family proteins in carcinogenesis and the treatment of cancer. Apoptosis 14: 584-596, 2009.

36. Scorrano L and Korsmeyer SJ: Mechanisms of cytochrome c release by proapoptotic BCL-2 family members. Biochem Biophys Res Commun 304: 437-444, 2003. 